\title{
Spububsaúde
}

\section{Incidência de diagnósticos de transtorno de personalidade: avaliação de adolescentes em acolhimento institucional identificados no serviço}

\author{
Incidence of personality disorder diagnoses: assessment of adolescents in institutional care \\ identified in the service
}

\author{
Guilherme Moura Gomes $^{1} \bullet$, Gleiciane Sabino Mateus Andrade ${ }^{2^{*}} \bullet$, Leonardo Ferreira Faria ${ }^{3} \bullet$
}

${ }^{1}$ Graduado em Psicologia, Especialista em Avalição Psicológica, Dalmass, Goiânia, Goiás, Brasil. ${ }^{2}$ Graduada em Fisioterapia, Especialista em Docência Universitária, Uniaraguaia, Goiânia, Goiás, Brasil. ${ }^{3}$ Docente, Mestrando em Ciências Criminológicas-Forenses, Especialista em Neuropsicologia, Criminologia e Psicologia Jurídica, Goiânia, Goiás, Brasil. *Autor para correspondência. E-mail:

gleicianemateus@gmail.com

\begin{abstract}
Resumo: Introdução: $\mathrm{O}$ acolhimento institucional de adolescentes sob medida protetiva de justiça traz em sua história adolescentes em situação de vulnerabilidades sociais os quais são colocados em abrigo provisório, a fim de garantir seus direitos básicos, para posteriormente reintegrá-los as famílias nuclear, extensa ou substituta. Objetivo: O objetivo da pesquisa é analisar a incidência de diagnósticos de transtorno de personalidade identificados no serviço. Materiais e métodos: Trata-se de um estudo documental, de caráter descritivo, com levantamento bibliográfico sobre a temática e abordagem qualitativa. Os sujeitos da pesquisa foram os documentos iniciais de admissão dos adolescentes em uma unidade de acolhimento e para a pesquisa bibliográfica foram utilizados os bancos de dados do Scientific Electronic Library Online (Scielo) e Biblioteca Virtual da saúde (BVS), do período de 2010 a 2020. Resultados e Discussões: Os resultados foram de 50 fichas com diagnósticos para transtorno de conduta e transtorno opositor desafiador. Considerações finais: Conclui-se que a incidência dos transtornos de personalidade é alta, mais evidente para o transtorno opositor desafiador e em maior número no sexo masculino.
\end{abstract}

Palavras-chaves: transtorno de conduta, transtorno de personalidade, jovem acolhido, criança institucionalizada, cuidados do lar adotivo.

\begin{abstract}
Introduction: the institutional care of adolescents under the protective measure of justice brings in its history adolescents in situations of social vulnerability who are placed in temporary shelter, in order to guarantee their basic rights, to later reintegrate them into nuclear, extended families or substitute. Objective: The objective of the research is to analyze the incidence of personality disorder diagnoses identified in the service. Materials and methods: This is a documentary, descriptive study, with a bibliographic survey on the subject and a qualitative approach. The research subjects were the initial documents for admission of the adolescents to a shelter unit and for the bibliographical research, the Scientific Electronic Library Online (Scielo) and Virtual Health Library (BVS) databases were used for the period 2010 to 2020. Results and Discussions: The results were 50 sheets with diagnoses for conduct disorder and oppositional defiant disorder. Final considerations: It is concluded that the incidence of personality disorders is high, more evident for oppositional defiant disorder, and higher in males.
\end{abstract}

Keywords: conduct disorder, personality disorder, sheltered youth, institutionalized child, foster home care.

\section{Introdução}

Os traços de personalidade das pessoas são diferentes e muitas vezes inflexíveis, dado a comprometimentos ou sofrimentos subjetivos que por diversos fatores podem resultar em má adaptação social. Os transtornos de personalidade podem ser influenciados por expressões e valores culturais, condições de saúde, padrões de vida e julgamentos sociais dos mais diversos tipos. Nesta perspectiva, os critérios para o diagnóstico são polêmicos e complexos, sendo heterogenia na prática, no entanto, são “compreendidos como transtorno mental, mas sugerindo-se mudanças nos critérios diagnósticos, arcabouços teóricos e métodos de avaliação e intervenção" (Soares, 2010).

Os transtornos de personalidade não estão presentes somente nos adultos, fazem parte também de grandes números envolvendo os adolescentes, principalmente os que estão em situações de vulnerabilidades. Assim, 
quando há a necessidade de acolhimento institucional, entende-se que o adolescente está em ambientes que não suprem suas necessidades básicas de vida, com ausência de apoio afetivo e exposição a violências psicológicas e/ou físicas (Silva et al., 2015).

De acordo com o Estatuto da Criança e do Adolescente (ECA) instituído pela Lei $\mathrm{n}^{\circ}$ 8.069/1990, as instituições de acolhimento existem para oferecer proteção em caráter provisório e excepcional a crianças e adolescentes que tiveram seus direitos violados ou ameaçados e cuja convivência com a família de origem seja considerada prejudicial ao seu desenvolvimento (Brasil, 1990).

Boa parte dos adolescentes em situações precárias, são diagnosticados com algum transtorno de personalidade, e nesta perspectiva os adolescentes são retirados de situações percebidas como de risco e de vulnerabilidades sociais, e são levados para abrigo provisório a fim de garantir seus direitos básicos como educação, lazer e saúde (Oliveira \& Milnitsky-Sapiro, 2007).

Estudos indicam que há aspectos positivos e de proteção presentes no processo de abrigamento. É visto que o serviço de institucionalização é capaz de oferecer melhores condições para os acolhidos, se configuram, muitas vezes, como a principal fonte de apoio e proteção para muitas crianças e adolescentes. No entanto outros autores, demonstram o quanto estereótipos negativos podem afetar o bem-estar e o ajustamento psicológico dos indivíduos, estando associados ao estresse, à depressão, ao medo, à baixa autoestima, entre outros (Wendt et al., 2017).

Carvalho et al. (2017), destacam que a permanência prolongada de crianças em instituições de abrigo pode fortalecer as chances de gerar patologias físicas e psíquicas, assim como déficits cognitivos. Entendendo que, mesmo o serviço garantindo os direitos básicos aos adolescentes, não é visto em primeiro momento como um local de segurança e que gera melhorias, isso porque há também altas chances de desencadear patologias e/ou fortalecimento de outras. Ideia reiterada por Gomide et al. (2017) quando afirmam que a permanência por longos períodos institucionalizados, sem intervenções especializadas, agrava potencialmente esses quadros.

Por este motivo é que se faz necessário o desenvolvimento de um serviço que esteja preparado para receber adolescentes em situações de vulnerabilidades que chegarão na instituição sob condições precárias, no que diz respeito a situação socioeconômica, mas também sobre a situação de saúde mental, exigindo do profissional, além do preparo, um olhar direcionado a situação social e que seja assertivo quanto aos motivos que levam àquele adolescente agir de maneira desajustada (Mendes, 2006).

É possível compreender, a incidência de diagnósticos pré-definidos anteriormente a algum dos contextos, seja ele protetivo ou socioeducativo, ambos demonstrando comportamentos desajustados dentro dos serviços em detrimento de uma estrutura familiar desajustada e falta de acompanhamento especializado ainda na infância ou na adolescência, na Tabela 1 estão os fatores de risco apresentados nesta faixa etária.

Tabela 1. Fatores de Riscos para criança e adolescentes.

\begin{tabular}{ll}
\hline & FATORES DE RISCOS \\
\hline Problemas de aprendizagem & Baixos níveis de afeto nas relações familiares \\
Baixa escolaridade & Associação a pares desviantes \\
Baixo monitoramento e ausência de regras & Histórico familiar infracional \\
Negligência familiar & Déficit de comportamentos morais \\
Nível sócio econômico reduzido & Genitores portadores de transtornos psicológicos \\
\hline
\end{tabular}

É importante frisar que a maior parte dos comportamentos apresentados pelos adolescentes, está restrito a questões sociais, ambientais e de estrutura familiar, dado que o reflexo da família nuclear diz respeito a saúde psicológica do indivíduo que ali se insere, ou seja, seu desajustamento psíquico está amplamente ligado à sua situação socioeconômica, sociocultural, ambiental e principalmente a situação afetiva (Guerra \& Prette, 2018).

Outro fator que deve ser considerado são os cuidados dos profissionais da equipe multidisciplinar que são indispensáveis neste contexto (Halpern et al., 2015), bem como a integração dos cuidados subjetivos, ou seja, como revela Carvalho et al. (2015) que este é "todo cuidado dispensado em função de um indivíduo em sua singularidade e que vai além do investimento voltado ao seu bem-estar físico”. Intervenções como essas é necessário para que o processo de socialização dos acolhidos seja maximizado quando comparado as situações vivenciadas anteriormente, de aspectos negativos e de descontrole familiar, e/ou até mesmo de violências de aspectos físicos e psicológicos. Visto que a instituição é uma proposta que o ambiente sociável da criança ou adolescente seja mais favorável ao bem-estar que o ambiente anterior em que se encontrava.

Neste cenário, surge os questionamentos: a institucionalização exacerba os diagnósticos mantendo-os de forma não investigada e pouco avaliativa ou busca por resultados de saúde fidedignos que levam a diagnósticos mais assertivos e ponderados levando em consideração a situação sociocultural e 
socioeconômica dos adolescentes? No entanto, também há a possibilidade de haver diagnósticos corretos e pouco avaliados, investigados ou tratados? São questionamentos necessários para o entendimento do crescente número de diagnósticos, sendo associados ou não a avaliações de forma superficial, com critérios pouco informativos ou descrevendo pouco envolvimento das situações e ambientes de vivência dos adolescentes, além dos comportamentos relatados por responsáveis e os clinicamente observáveis por profissionais de saúde ou se de fato são adolescentes que apresentam transtorno de personalidade.

A principal motivação para a realização da pesquisa é dada pela importância de compreender todas as condições socioeconômicos e socioeducativos do qual o adolescente estava inserido antes do processo de institucionalização. Pois, pode-se afirmar que esses fatores contribuem para construção da estrutura familiar, e assim consequentemente para a formação da personalidade e no comportamento dos adolescentes. Desta maneira, busca-se o entendimento dos fatores que influenciam na tomada de decisão quanto ao diagnóstico de transtorno de personalidade, e a respeito da atuação dos profissionais de saúde envolvidos no processo de garantia dos direitos básicos como de saúde, educação, lazer, entre outros.

Assim, o objetivo da pesquisa é analisar a incidência de diagnósticos de transtorno de personalidade identificados no serviço, além da possível estimulação da potencialização de atitudes e comportamento dos acolhidos devido insuficiência de análise do perfil social realizada pelos profissionais de saúde influenciando na tomada de decisão quanto ao diagnóstico antes da institucionalização.

\section{Material e métodos}

Trata-se de um estudo documental, de caráter descritivo, com levantamento bibliográfico sobre a temática e abordagem qualitativa. Os sujeitos da pesquisa foram os documentos iniciais de admissão dos adolescentes em uma unidade de acolhimento para adolescentes da região metropolitana de Goiânia, que tem por finalidade abrigar adolescentes de 12 a 17 anos e 11 meses em situação de vulnerabilidade social.

Foram utilizados documentos institucionais acerca dos adolescentes com registros na instituição, baseando em relatórios iniciais da equipe técnica, como fonte primária de dados, ou seja, sem contato com qualquer indivíduo para a realização desta pesquisa, em complemento, como fonte de dados secundários, foi realizado o levantamento bibliográfico relacionado ao tema, que discutem a institucionalização e seus processos.

Os dados observados foram dispostos em forma descritiva e em gráficos. Para a pesquisa bibliográfica foram utilizados os descritores de saúde: transtorno de conduta, transtorno de personalidade, jovem acolhido, criança institucionalizada, cuidados do lar adotivo. Nos bancos de dados do Scientific Electronic Library Online (Scielo) e Biblioteca Virtual da saúde (BVS), do período de 2010 a 2020, em português, somente textos completos e gratuitos, foram exclusos os materiais que não apresentaram características referentes a base principal do estudo. Por fim, os achados é parte do processo de discussão. Salienta-se que não houve nenhuma limitação durante a pesquisa e nem conflitos de interesses.

\section{Resultados e discussão}

De acordo com os documentos dispostos para a análise de 4 anos de funcionamento da instituição, uma unidade de acolhimento para adolescentes da região metropolitana de Goiânia, foram achados 50 relatórios dos quais apenas 15 possuíam diagnóstico referentes a transtorno de personalidade: Transtorno Opositor Desafiador (TOD) e Transtorno de Conduta (TC).

Desses, somente 1(um) dos diagnósticos foi realizado pelo psicólogo, enquanto aos demais (14 diagnósticos) foram realizados pelo médico psiquiatra. Observa-se que quando se refere a parcela de adolescentes, quanto ao gênero, sobressai-se os do sexo masculino (Figura 1). E quanto as idades dos adolescentes, houve a variação entre 14 a 17 anos (Figura 2).

Notou-se que a prevalência de diagnósticos foi identificada o TOD, seguido do TC (Figura 3), outro fator analisado foi o tempo de acolhimento que sofreu variações de 1 (um) mês há 3 anos de institucionalização (Figura 4).

Os gráficos mostram ilustrativamente as prevalências e caracterização dos adolescentes na instituição, aspectos necessários para o processo de acolhimento, especialmente na anamnese. 


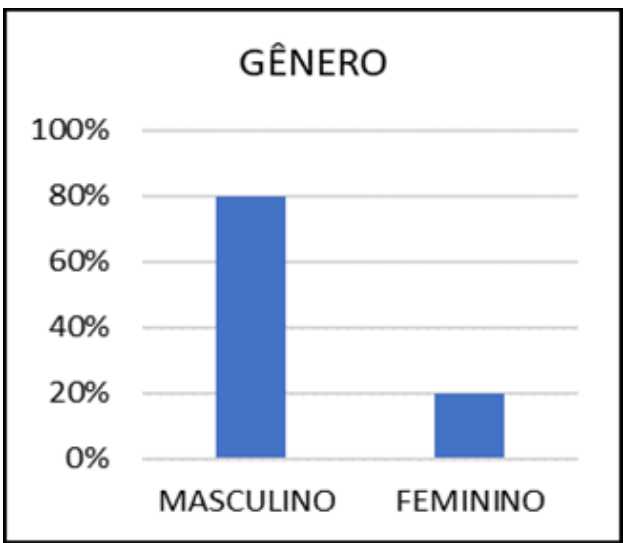

Figura 1. Variação quanto ao gênero. Fonte: Autor (2020).

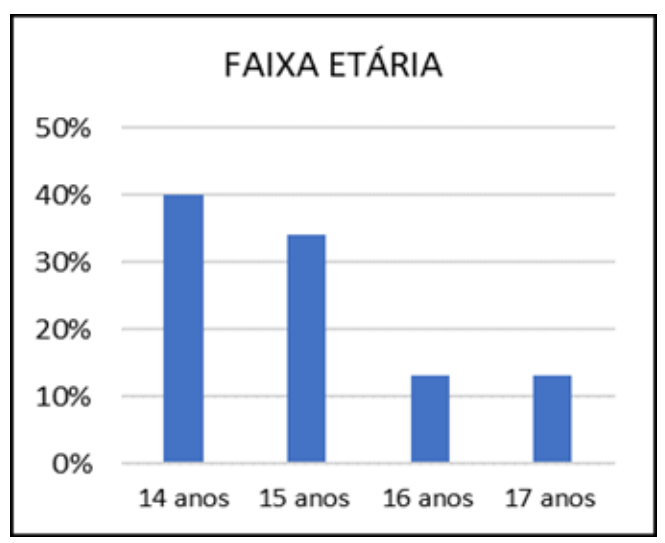

Figura 2. Variação de idade dos adolescentes na instituição. Fonte: Autor (2020).

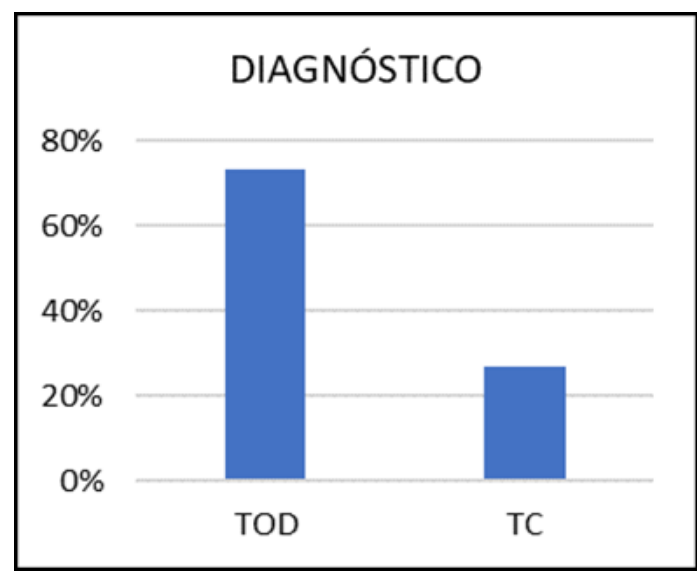

Figura 3. Variação de diagnóstico dos adolescentes. Fonte: Autor (2020).

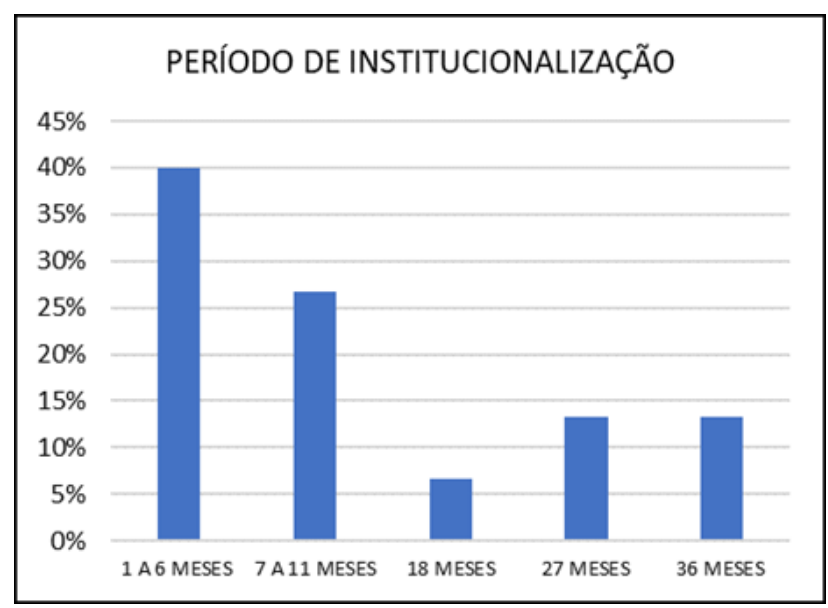

Figura 4. Período de Institucionalização dos adolescentes. Fonte: Autor (2020). 
Acredita-se que o processo de avaliação para que o adolescente seja encaminhado para a instituição, ainda percorre a necessidade de considerar de forma minuciosa aspectos que envolve o perfil social dos adolescentes, pois compreende-se que é um fator condicionante das experiências dos indivíduos, podendo refletir no comportamento e atitudes. Outra condição importante é o processo de reavaliação realizado pelos profissionais de saúde que visa sempre abranger de forma integral a vivência e fatores que dispôs os comportamentos dos adolescentes, visto que mesmo com melhoras, mediante os retornos no psicólogo e a medicalização, devem ser sempre observados para que não haja recidivas comportamentais.

Para Novais (2016), as modificações que acontecem no mundo interno de um adolescente, incluindo a construção do pensamento, é o início de "encontrar-se" em meio a sociedade, mesmo com poucas experiências, estes tentam a solução de conflitos e recriações de situações vivenciadas contribuindo para a sensação de recuperação do controle ao seu redor. Alega-se que a dificuldade neste contexto, é o manejo para os diagnósticos nesta fase, pois trata-se de um período de instabilidades emocionais, psicológicas e físicas próprias da fase. Apontando que, a relevância nesses casos é a de identificar o que é um comportamento típico da adolescência para manifestações psicopatológicas.

Concomitantemente, Vilhena e Paula (2017) destacam que o TC é caracterizado como padrão repetitivo e persistente, apresentando dificuldades de autocontrole, consistindo em comportamentos que violam as 'regras' sociais. O destaque é para o sexo masculino, geralmente expostos em situações em ambientes desfavoráveis gerando carências e dificuldades de lidar com as emoções, tal como desrespeito com 'o outro', abrangendo também, na grande maioria, a violência. Desse modo, em relação ao outro transtorno, o TOD, que para Almeida et al. (2014), é de aspecto recorrente, do comportamento negativista, desafiador e hostil, envolvendo a desobediência com figuras de autoridade, além da agitação e recusas a solicitações e irritações frequentes, entre outros aspectos. Comportamento que similarmente se mostrou mais frequente em crianças e adolescentes do sexo masculino.

A princípio, alguns estudos relatam que não há muitas diferenças encontradas nas práticas familiares quanto aos cuidados do adolescente em situações de vulnerabilidades a respeito da situação de gênero, esta não é uma questão que desperte preocupações nos pais, ou seja, não foi identificado preocupações relevantes ou demasiadas, quando se trata somente de questões envolvendo os gêneros (feminino ou masculino). No entanto, durante o período de acolhimento há a necessidade de compreender as questões de cada gênero, fatores educativos, físicos e emocionais, pois cada fase ocorre distinções e alterações conforme o sexo e idade, estas por sua vez devem ser pautadas nos acompanhamentos realizados pelos profissionais de saúde, traçando as práticas e habilidades que possam influenciar no crescimento pessoal saudável de cada indivíduo (Sabbag \& Bolsoni-Silva, 2011). Segundo os aspectos que envolve a faixa etária dos adolescentes, de acordo com a pesquisa de Fernandes e Monteiro (2017), a prevalência esteve entre as idades de 15 a 18 anos, o mesmo foi observado como predominância no gráfico 2.

Outra peculiaridade observada foi referente ao tempo de institucionalização, bem como mostrado no gráfico 4, concluiu-se que em muitas vezes, o período pode ultrapassar dois anos, condições influenciadas por diversos aspectos. Em concordância, Acioli et al. (2013) acrescenta destacando que o sexo masculino é susceptível há necessitar de mais tempo na instituição, fatores geralmente relacionados ao comportamento violento ou ainda por apresentarem maior número de evasões retratadas no processo de institucionalização.

Em complemento, o Estatuto da Criança e Adolescente destaca o caráter provisório do acolhimento institucional, o que na prática, é demonstrado erroneamente, vez que existem acolhimentos que ultrapassam o tempo de dois anos (Brasil, 1990), fazendo com que aquele acolhido veja a instituição como sendo sua casa, ou em virtude de comportamentos desajustados, rebele-se contra a mesma e, de maneira consciente, aja com atos infracionais.

Diversos autores apontam questionamentos acerca da institucionalização e o agravamento desta no que diz respeito ao desenvolvimento cognitivo e até psíquico de adolescentes em situação de vulnerabilidade e consequentemente do acolhimento, podendo identificar a existência de comportamentos vistos como desajustados e, como avaliado a partir da observação feita in loco, tidos como diagnósticos concretos de algum tipo de transtorno de personalidade (Fonseca, 2017).

Gomide et al. (2014), diferente de Fernandes et al. (2015), reitera que os adolescentes já apresentam alguns comportamentos como sendo característicos deles anteriormente à situação de acolhimento, devido as condições ambientais, socioeconômicas, estruturais, pois, já são tomados por experiências negativas e estressoras desde a primeira infância e ensinados àquelas práticas desajustadas, vez que é possível identificar no contexto familiar tais comportamentos agressivos, opositores, de falta de afeto, desafiantes, dentre outros.

Carvalho et al. (2017) e Gomide et al. (2017), reforçam que a institucionalização que pode durar, como já mencionado, mais de dois anos, agrava estes comportamentos inadequados e fortalece patologias psíquicas 
que não houveram intervenções por equipe especializada. Em campo é possível perceber a incidência destes perfis alterados e desajustados quando eles fazem o primeiro contato com a instituição, os adolescentes agem infringindo regras institucionais cometendo atos infracionais, desestabilizando os demais acolhidos, não aderindo aos programas oferecidos, dentre outras práticas, a partir de sua permanência e após as intervenções dos profissionais, ali capacitados, começam a compreender o funcionamento institucional e os seus princípios norteadores, possibilitando que eles tenham acesso aos seus direitos básicos, dentre eles, educação, lazer e saúde.

Fernandes et al.(2015) e Acioli et al. (2013), apresentam a ideia que a situação de acolhimento como desencadeante de transtornos e demais déficits, sendo possível de identificar diagnósticos mais evidentes nas instituições e não somente nas famílias de origem dos adolescentes institucionalizados, o que pode ser visto como uma falha no atendimentos próprios da saúde mental na infância, vez que não busca orientar as famílias, pois na maior parte, se trata de famílias de baixa renda que não compreendem comportamentos desajustados como necessários de intervenção psicológica ou psiquiátrica, vendo-os apenas como comportamentos comuns da infância e não possuindo acesso aos serviços disponibilizados.

Em contrapartida, Wendt et al. (2017), Guerra e Prette (2018), entendem o papel do acolhimento como sendo uma possibilidade de apoio e intervenção aos adolescentes institucionalizados, o que pode ser observado nas evoluções da equipe técnica presente na instituição, sendo que adolescentes já chegam na instituição com comportamentos opositores, desafiadores e ameaçadores, não apenas por questões sociais de desvinculação da família, mas também apresentando, após o acolhimento, comportamentos característicos de sua personalidade devido toda a situação social, socioeconômica e socioambiental em que estavam inseridos desde a infância, não possuindo qualquer auxílio referente a saúde mental, passando a possuir na instituição os serviços de apoio e atendimentos necessários que deem vasão a melhora da condição psicológica que apresentam.

Neste contexto, notou-se que o acolhimento possibilitou melhora nos comportamentos impulsivos, hostis e pouco sociais, ademais é importante acrescentar que toda a equipe multidisciplinar trabalha em conjunto realizando acompanhamento de caráter especifico e minucioso, conduzindo com frequência semanal os atendimentos psicológicos, fomentando os retornos ao médico psiquiátrico entre outras ações simultâneas. Para Carvalho et al. (2015) a influência da institucionalização também é positiva, pois quando o ambiente familiar é extremamente desordenado, o adolescente em fase inicial pode opor-se a regras, mas com o tempo e a visualização do comportamento dos profissionais e colegas, bem como troca de experiências, sentimentos, valores e percepção de novos significados em diversas situações, podem se deixar influenciar nos comportamentos e auxiliar no enfrentamento de situações do cotidiano. Visto que é destacado por Lima (2018) e Teixeira (2014), a instituição passa a ser o espaço social do adolescente e nele sua experiência social.

Dentre os fatores observados, considera-se que durante o processo de acolhimento muitos adolescentes apresentam disponibilidade e aceitação de intervenções. Bem como alega Martins (2012) e Souza et al. (2018), há certa compreensão por parte dos acolhidos que o processo de institucionalização é uma oportunidade de recomeço e formação de novas ideologias, pensamento apresentado geralmente durante o questionamento e intervenções mediante a reflexão de futuro. Mesmo por apresentarem reflexão superficial sobre as mudanças necessárias para a vivência em sociedade, ver-se que categoricamente há estimulo básicos para a estrutura reflexiva sobre contextos de responsabilidades consigo e sobre a comunidade inserida, marcando aspectos de crescimento individual dos adolescentes e nas ações positivas no comportamento mesmo em circunstâncias de institucionalização.

Destaca-se as questões apresentados por Silveira et al. (2015), demonstrando os diversos comportamentos e atitudes desajustadas oriundas de aspecto social que devem ser minuciosamente analisadas, pois corrobora com a incidência de adolescentes que apresentam comportamentos desafiadores, opositores e de conduta devido a situação familiar desestruturada.

Ainda no cenário socioeducativo, percebe-se que neste caso os adolescentes em sua maioria com diagnósticos já definidos devido suas condutas e atos infracionais graves cometidos na adolescência, são eles, portanto, vítimas de um sistema que não consegue amparar todos aqueles que, de alguma forma demonstram precisar de apoio, isso valendo para as famílias e também para estes adolescentes que já possuíam algum desajustamento psicológico (Zappe \& Dell'aglio, 2016).

Esses fatores para Gabatz et al. (2010), quando reconhecidos ainda na infância diminui-se os efeitos deletérios que podem ser causados às crianças e adolescentes. Aspectos destacado também por Jordão e Ramires (2010), do que diz a respeito da família, entender-se que a sociedade contemporânea em geral, pode estar sendo potencializada com questões que destacam as individualidades e indiferenciações entre as gerações, e os frágeis vínculos afetivos estimulando o sofrimento psíquico dos adolescentes, questões que necessitam de discussão e investigação mais detalhadas. 


\section{Considerações finais}

A incidência dos casos de transtornos de personalidade em maior número foi referente ao TOD, e maior ocorrência no sexo masculino, entre a faixa etária de 14 anos, em seguida a idade de 15 anos, tendo o período de institucionalização de 1 a 6 meses, sucessivamente, o período de 7 a 11 meses, com ocorrências de até 36 meses de institucionalização.

De acordo com os achados, foi contatado que não é determinante a situação de acolhimento ou o tempo que eles estão acolhidos que estimulam a incidência de comportamentos compatíveis com transtornos de personalidade ou outros, mas sim as vivências e as situações anteriores ao acolhimento, vez que estão expostos a condições, muitas vezes, subumanas, não tendo de sua família nuclear qualquer intenção ou interação afetuosa, de cuidado, já sendo possível verificar que esta mesma família possui outros desajustes como transtornos e doenças diversas, o que também pode acarretar na falta desses comportamentos afetuosos e cuidadosos que deveriam ser típicos da família nuclear.

Considerou-se também, que a institucionalização é colocada como um fator de estimulação destes comportamentos desajustados, uma vez que existem no contexto inúmeros adolescentes que possuem atos infracionais ou que os cometem durante a institucionalização, não sendo a institucionalização o fator predominante desses atos e sendo eles ligados aos transtornos, muitos desses como os de personalidade, sejam TC e TOD, e outros.

É possível afirmar que os comportamentos desajustados que vão de encontro aos transtornos de personalidade não são estimulados, evoluídos ou criados por conta da situação de acolhimento, uma vez que toda a experiência anterior do adolescente é favorável para a estimulação destes, sendo na primeira infância que estes conflitos se destacam na família nuclear, e assim, vistos desde sempre como comuns.

Durante o tempo em que os adolescentes estão no serviço de acolhimento eles passam por diversos serviços de saúde, contando com profissionais capacitados nos serviços que contribuem para a estabilização de comportamentos impulsivos, déficits, transtornos variados e auxiliam na diminuição destes atos, sendo, portanto, a unidade de acolhimento um forte apoio para os adolescentes contribuindo para o melhor desenvolvimento pessoal e coletivo.

Mediante as observações apresentadas, há sem dúvidas, carência de melhores explorações sobre a temática, visando a possibilidade no contexto de avaliação prática para o processo de institucionalização desses adolescentes, sob melhores condições de manejo a respeito das necessidades desta população frentes aos serviços garantidos em lei.

\section{Referência}

Acioli, R. M. L., Barreira, A. K., Lima, M. L. C., Assis, S. G., \& Lima, M. L. L. T. 2019. Tempo de acolhimento e características dos adolescentes acolhidos por tipo de serviços institucionais. Recife, Brasil, 2009-2013. Ciência \& Saúde Coletiva, 24(2); 553-62.

Almeida, L. R. P., Ferreira, L. V. G., Zamarchi, P. F., Alves, D. R. M., Xavier, R. N., \& Boni, E. P. P. 2014. Análise funcional em um estudo de caso de transtorno desafiador de oposição e transtorno de conduta. Revista Científica da Faculdade de Educação e Meio Ambiente, 5(2);15-35.

Brasil. 1990. Lei no 8.069, de 13 de julho 1990. Dispõe sobre o Estatuto da Criança e do Adolescente e dá outras providências. Disponível em: www.planalto.gov.br/ccivil_03/LEIS/L8069.htm\#art267

Carvalho, C. F., Razera, J., Haack, K. R., \& Falcke, D. 2015. Acolhimento institucional: considerações sobre a forma como o cuidado subjetivo se apresenta no cotidiano de trabalho dos educadores sociais. Aletheia, (47-48), 51-63.

Carvalho, M. I., Santos, R. G. M., \& Cavalcante, D. M. 2017. Os Impactos Da Institucionalização no Desenvolvimento Cognitivo. Revista de Iniciação Científica da Ulbra, (15).

Fernandes, A. O., Oliveira-Monteiro, N. R., Spadari-Bratfisch, R. C., Nascimento, J. O. G., \& Montesano, F. T. 2015. Estresse em adolescentes abrigados. Adolescencia e Saude, 12(4), 65-75.

Fernandes, A. O., \& Monteiro, N. R. D. O. 2017. Comportamentos Pró-Sociais de Adolescentes em Acolhimento Institucional1. Psicologia: teoria e pesquisa, 33.

Fonseca, P. N. 2017. O impacto do acolhimento institucional na vida de adolescentes. Revista de Psicopedagogia, 34(105);285-96.

Gabatz, R. I. B., Padoin, S. M. D. M., Neves, E. T., \& Terra, M. G. 2010. Fatores relacionados à institucionalização: perspectivas de crianças vítimas de violência intrafamiliar. Revista Gaúcha de Enfermagem, 31, 670-677. 
Gomide, P. I. C., Mascarenhas, A. B. D., \& Munhoz, G. V. 2017. Avaliação de uma intervenção para redução de comportamentos antissociais e aumento da escolarizaçãoem adolescentes de uma instituição de acolhimento. Acta Comportamentalia: Revista Latina de Análisis de Comportamiento, 25(1), 25-40.

Guerra, L. L., \& Prette, Z. P. 2018. Habilidades sociais educativas de cuidadores de crianças institucionalizadas. Arquivos Brasileiros de Psicologia, 70(3), 98-112.

Halpern, E. E., Leite, L. M. C., \& Moraes, M. C. M. B. (2015). Seleção, capacitação e formação da equipe de profissionais dos abrigos: o hiato entre o prescrito e o real. Trabalho, Educação e Saúde, 13, 91-113.

Jordão, A. B., \& Ramires, V. R. R. 2010. Adolescência e organização de personalidade borderline: caracterização dos vínculos afetivos. Paidéia, 20(47), 421-430.

Mendes, P. M. 2006. A doutrina da proteção integral da criança e do adolescente frente à Lei 8,069/90. Dissertação de mestrado em Direito Difusos e Coletivos. São Paulo, SP: Pontifícia Universidade Católica de São Paulo.

Novais, G. R., Cordoni, J. K., Reato, L. F. N., \& Marinho, M. S. 2016. Transtorno de conduta na adolescência: recortes de um caso clínico. inFormação, 20(20), 49-60.

Sabbag, G. M., \& Bolsoni-Silva, A. T. 2011. A relação das Habilidades Sociais educativas e das práticas educativas maternas com os problemas de comportamento em adolescentes. Estudos e Pesquisas em Psicologia, 11(2), 423-441.

Silva, C. D. L., Denardi, R. C., Becker, A. P. S., \& Silva Delvan, J. 2015. A Psicologia nos serviços de acolhimento institucional e o fortalecimento de vínculos familiares e comunitários. Revista Pesquisas e Práticas Psicossociais, 10(1), 55-65.

Silveira, K. S. S., Zappe, J. G., \& Dias, A. C. G. 2015. Correlatos dos comportamentos antissociais limitados à adolescência e dos comportamentos antissociais persistentes. Psicologia em Estudo, 20(3),425-436.

Soares, M. H. 2010. Estudos sobre transtornos de personalidade Antissocial e Borderline. Acta Paulista de Enfermagem, 23, 852-858.

Teixeira, L. C. 2014. O sujeito adolescente e a intervenção psicanalítica: notas a partir de um caso clínico. Revista Latinoamericana de Psicopatologia Fundamental, 17, 797-804.

Vilhena, K., \& Paula, C. S. D. 2017. Problemas de conducta: prevalencia, factores de riesgo/protección y el impacto en la vida escolar y la edad adulta. Cadernos de Pós-Graduação em Distúrbios do Desenvolvimento, 17(1), 39-52.

Wendt, B., Dullius, L., \& Dell'aglio, D. D. 2017. Imagens Sociais sobre Jovens em Escolha Institucional. Psicologia Ciência e Profissão, 37(2), 529-541.

Zappe, G. J., \& Dell'aglio, D. D. 2016. Risco e proteção no desenvolvimento de adolescentes que vivem em diferentes contextos: família e institucionalização. Revista Colombiana de Psicologia. 25(2),289-305.

\section{Minicurrículo}

Guilherme Moura Gomes. Psicólogo Clínico, Especialista em Avaliação Psicológica pela Dalmass Cursos, DALMASS, Brasil.

Gleiciane Sabino Mateus Andrade. Fisioterapeuta, Especialista em Docência Universitária pela Uniaraguaia, Brasil.

Leonardo Ferreira Faria. Docente, Mestrando em Ciências Criminológicas-Forenses, Especialista em Neuropsicologia, Criminologia e Psicologia Jurídica. Psicólogo Criminal da Polícia Técnico-Científica do Estado de Goiás.

Como citar: Gomes, G.M., Andrade, G.S.M., \& Faria, L.F. 2021. Incidência de diagnósticos de transtorno de personalidade: avaliação de adolescentes em acolhimento institucional identificados no serviço. Pubsaúde, 7, a224. DOI: https://dx.doi.org/10.31533/pubsaude7.a224

Recebido: 8 jul. 2021.

Revisado e aceito: 21 jul. 2021.

Conflito de interesse: os autores declaram, em relação aos produtos e companhias descritos nesse artigo, não ter interesses associativos, comerciais, de propriedade ou financeiros que representem conflito de interesse.

Licenciamento: Este artigo é publicado na modalidade Acesso Aberto sob a licença Creative Commons Atribuição 4.0 (CC-BY 4.0). 